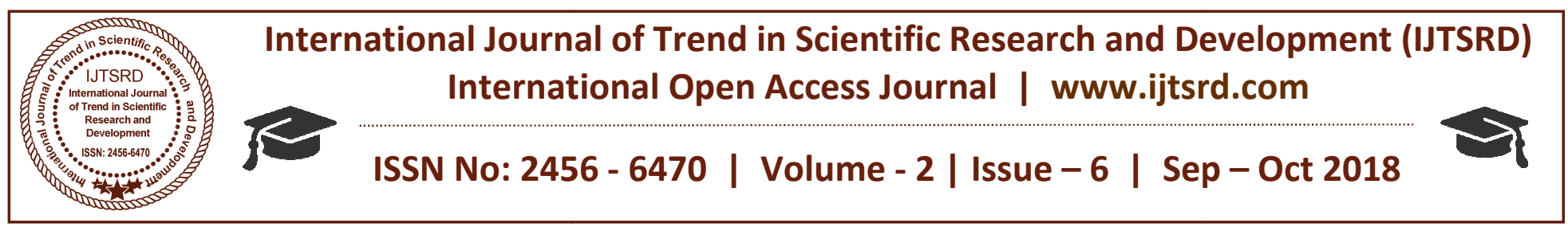

\title{
Performance Improvement of QCA Design XOR Logic Gate using Bistable Simulation Engine Vector
}

\author{
Haritika Satle, Aastha Hajari \\ Embedded System and VLSI Design, Department of Electronics and Communication Engineering, \\ Shiv Kumar Singh Institute of Technology \& Science (SKSITS), Indore, Madhya Pradesh, India
}

\begin{abstract}
Among the emerging technologies recently proposed as alternatives to the CMOS technology, the quantumdot cellular automata is one of the promising solutions to design very high speed and ultralow power digital circuits. In this paper we used QCA Designer simulator tool for simulation of the proposed XOR design. The QCA Designer tools used to simulate the circuits and calculate the area as well as number of cells.
\end{abstract}

Keyword: QCA, XOR, Majority Gate, QCA Cells, Bistable Simulation Engine.

\section{INTRODUCTION}

QCA is a computational nanotechnology that can be used to construct nano-scale circuits. Nowadays, this technology is a popular alternative for CMOS technology due to features such as speed, low occupied area and low power consumption [1]. The many technologies have been investigated such as Carbon Nano Tube field effect transistors (CNTFETs), Single electron transistors (SETs), Quantum-dot cellular automata (QCA) and others. QCA is a promising technology supporting a transistor-less paradigm. This QCA technology has quantum cells. The quantum cells has 2 electrons present at a time, has 4 quantum wells and 2 electrons are occupies in adjacent position to each other. The rest of paper is organised in section A.1 is QCA building blocks, II-Section QCA clock diagram dissection, Section-III Simulation design and results and last section IV is conclusion and references at the last of paper.

\section{A. QCA building blocks}

The building blocks are determined by the fundamental logic gate structure. Each QCA cell contains two electrons and four quantum dots and due to Coulomb interaction between these identical charges, they occupy dots diagonally. As a result, the two stable polarization states for a QCA cell are achieved, as shown in Figure 1. The instantaneous polarization of a cell is denoted as either -1 or +1 [2], which are encoded to represent a binary " 1 " value and " 0 " value, respectively. In order to create any digital logic, basic elements including wire, NOT gate, AND gate, OR gate and majority gate are required to implement that logic $[1,3]$. Since no electrons tunnel between cells, QCA provides a mechanism for transferring information without current flow [6].

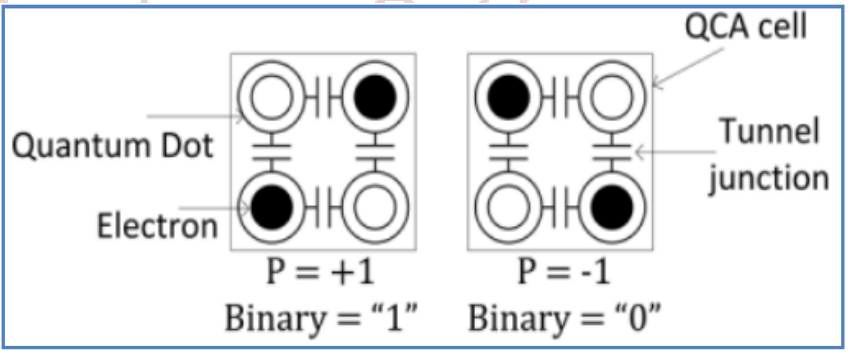

Fig.1: Basic QCA cell with two binary state [7]

The Majority voter (M), five QCA cells that realize the function of in equation 1, and its QCA design is shown in figure 2.

$$
M(a, b, c)=a b+b c+a c
$$

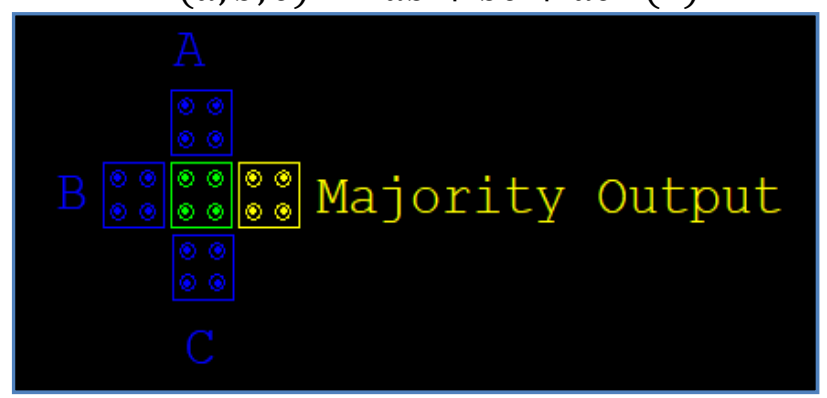

Fig. 2: MV QCA Design 
For example, a two-input AND gate is realized by fixing one of the majority gate inputs to " 0 ," that is in equation 2 ,

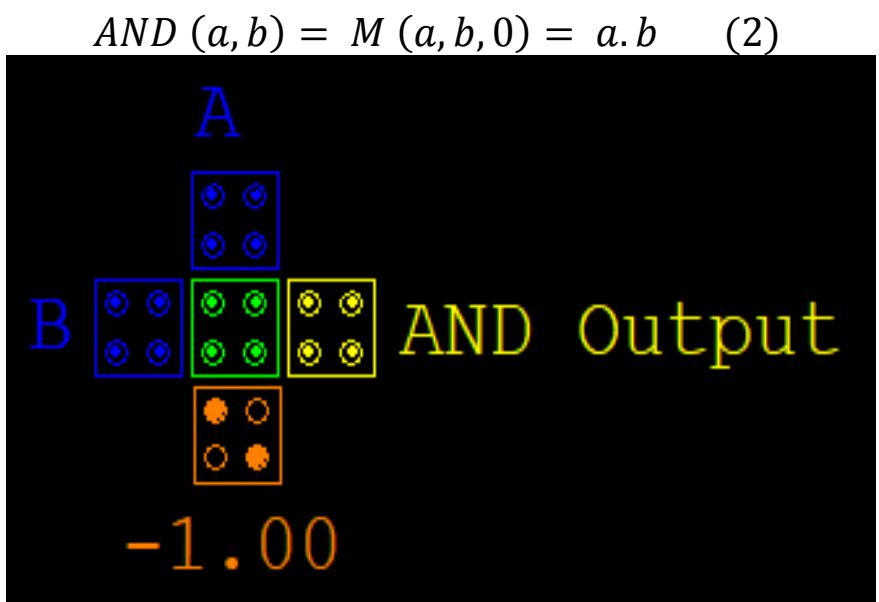

Fig. 3: AND Logic Gate using QCA Cells

Similarly, an OR gate is realized by fixing one input to " 1 ," that is in equation 3 ,

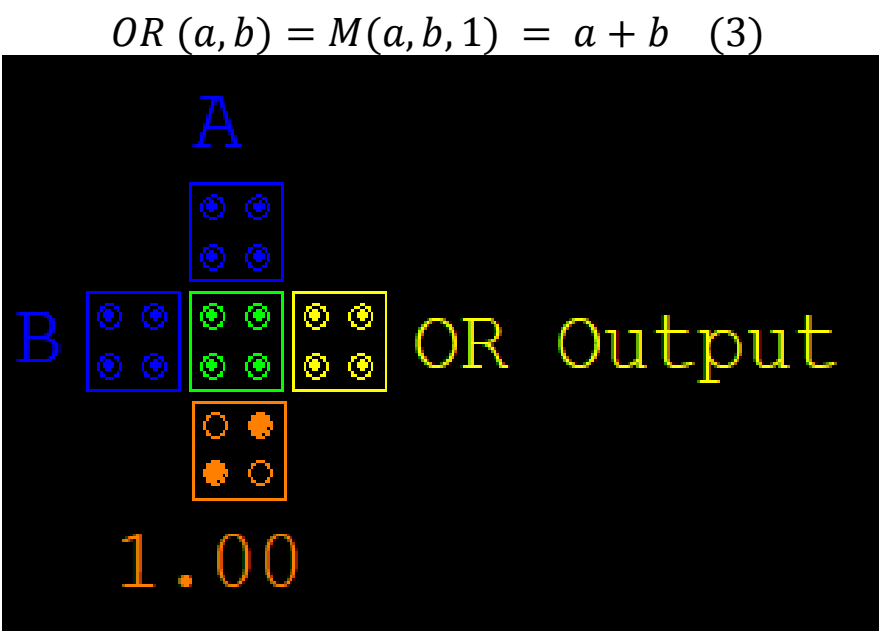

Fig. 4: QCA OR Logic Gate

\section{QCA CLOCK}

In QCA technology, storage cells do not require an external power source to maintain their current stable polarization. Actually, the clock controls the flow of charge in the circuit. The QCA clock consists of four clock phases, i.e. Switch, Hold, Release, and Relax, which span a 90 degree out-of phase progression [4, 5]. It is shown in figure 5 for QCA clock diagram. This diagram $\mathrm{X}$-axis represents the time and $\mathrm{Y}$-axis represents the inter-dot-barrier.

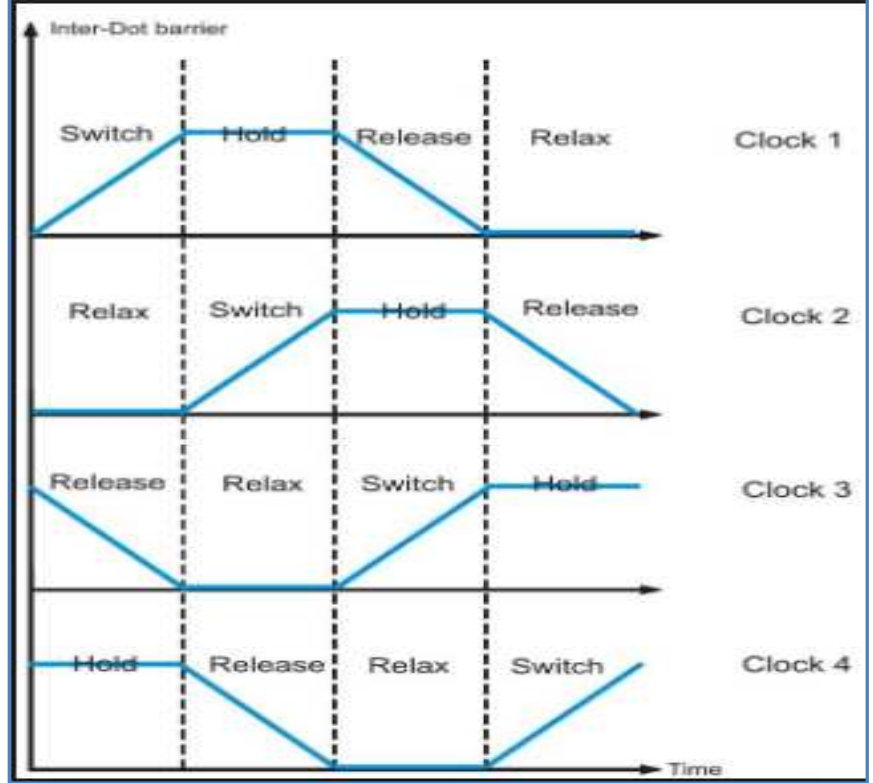

Fig. 5: QCA Clock [7]

\section{SIMULATION RESULTS}

We are proposed two different QCA "XOR logic design" in figure 4.5 XOR-1 with using Quantum cells 27 used design area in $0.04 \mu \mathrm{m}^{2}$ with utilized single layer design and clock cycle delay count one. This design is efficient in team of design cell area as well as used cell count.

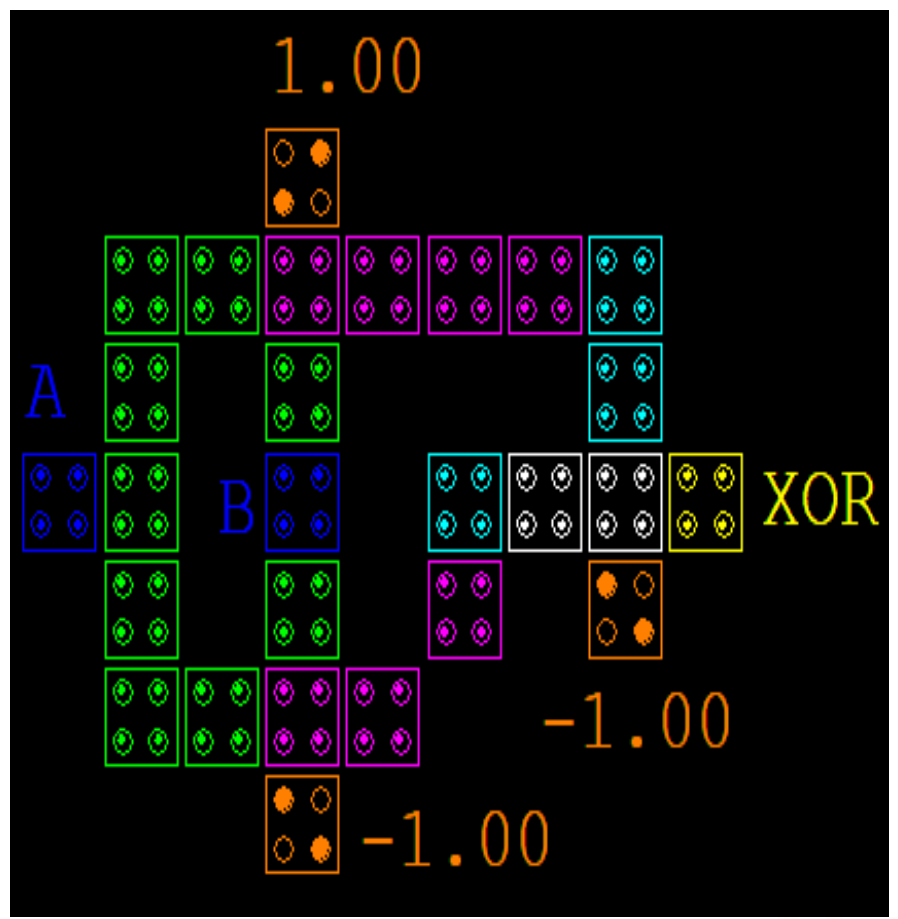

Fig. 6: Proposed QCA-XOR Logic Gate Design 


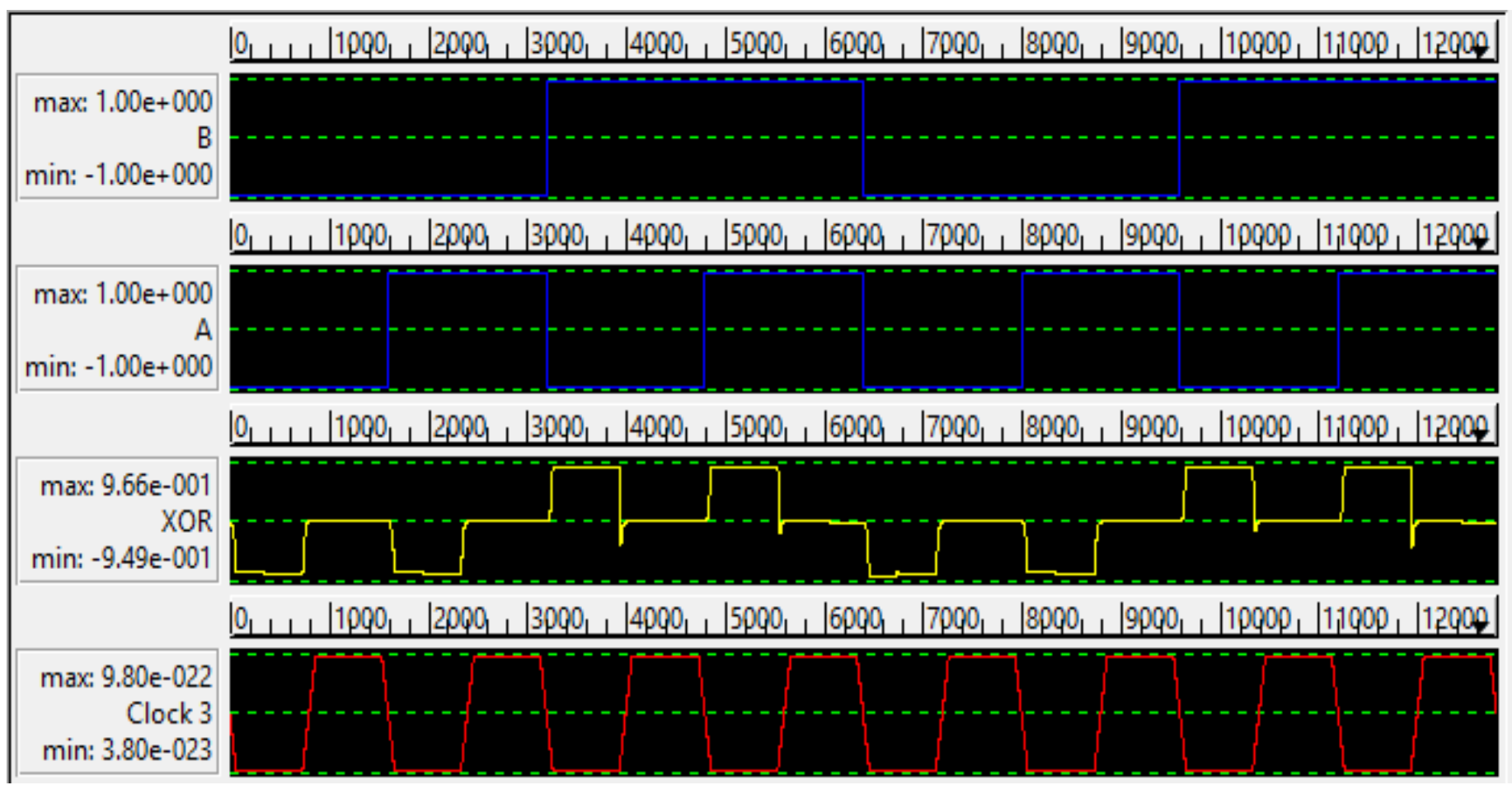

Fig. 7: Simulation of XOR Logic Gate

Table 1: Comparison of Proposed Design and Previous design of QCA XOR

\begin{tabular}{|c|c|c|c|}
\hline Design & Cell & Area & Delay \\
\hline Proposed Design XOR & 27 & 0.04 & 1 \\
\hline Mrinal Goswami (2014) et al. [9] & 40 & 0.04 & 3 \\
\hline Young-Won You (2016) et al. [10] & 44 & 0.05 & 4 \\
\hline
\end{tabular}

\section{CONCLUSIN}

CMOS technology is approaching its scaling limit very fast. Quantum-dot Cellular Automata (QCA) is an emerging nanotechnology, with extremely small feature size and ultra low power consumption compared to transistor-based technology. The proposed design results analysis is shown in table 1 in this table we compared the both design XOR logic gate on the bases of quantum cells, design area and delay. The proposed design used the bistable simulation engine.

\section{REFRENCES}

1. Javad Chaharlang, Mohammad Mosleh "An overview on RAM memories in QCA technology" Majlesi Journal of Electrical Engineering, Vol. 11(2), 2017.

2. G. Cocorullo, P. Corsonello, F. Frustaci and S. Perri "Design of Efficient BCD Adders in Quantum-Dot Cellular Automata" IEEE tns on Circuits and Systems II, Vol. 64(5), 2017.
3. Shanthala.G.M, Riazini and Karthik.P "Design and Implementation of Scan Flip-flop for Processor Using QCA Technology" International Journal of Control and Automation Vol.10, No.8 (2017), pp.41-52.

4. S. Sheikhfaal, S. Angizi, S. Sarmadi, M. H. Moaiyeri, and S. Sayedsalehi, "Designing efficient QCA logical circuits with power dissipation analysis," Microelectronics Journal, vol. 46, pp. 462-471, 2015.

5. S. Srivastava, S. Sarkar, and S. Bhanja, "Estimation of upper bound of power dissipation in QCA circuits," Nanotechnology, IEEE transactions on, vol. 8, pp. 116-127, 2009

6. Fenghui Yao, Mohamed Saleh Zein-Sabatto, Guifeng Shao, Mohammad Bodruzzaman, and Mohan Malkani "Nanosensor Data Processor in Quantum-Dot Cellular Automata" Journal of Nanotechnology Vol. 2014, Article ID 259869, 14 pages 
7. Patidar M., Gupta N. (2019) Efficient Design and Simulation of Novel Exclusive-OR Gate Based on Nanoelectronics Using Quantum-Dot Cellular Automata. In: Nath V., Mandal J. (eds) Proceeding of the Second International Conference on Microelectronics, Computing \& Communication Systems (MCCS 2017). Lecture Notes in Electrical Engineering, vol 476. Springer, Singapore.

8. Esam Alkaldy, Keivan Navi "Reliability Study of Single Stage Multi-Input Majority Function for QCA" International Journal of Computer Applications, Volume 8(3), 2013.
9. Goswami, M., Kumar, B., Tibrewal, H., \& Mazumdar, S. (2014). Efficient realization of digital logic circuit using QCA multiplexer. 2014 2nd International Conference on Business and Information Management (ICBIM).

10. Young-Won You and Jan-Chelo Jeon (2016), Coplanar wire crossing based QCA XOR Gate using NAND, NOR invertors gate, Asia-pacific proceedings of applied science and engineering for batter human life, Vol. 6, pp. 41-44.

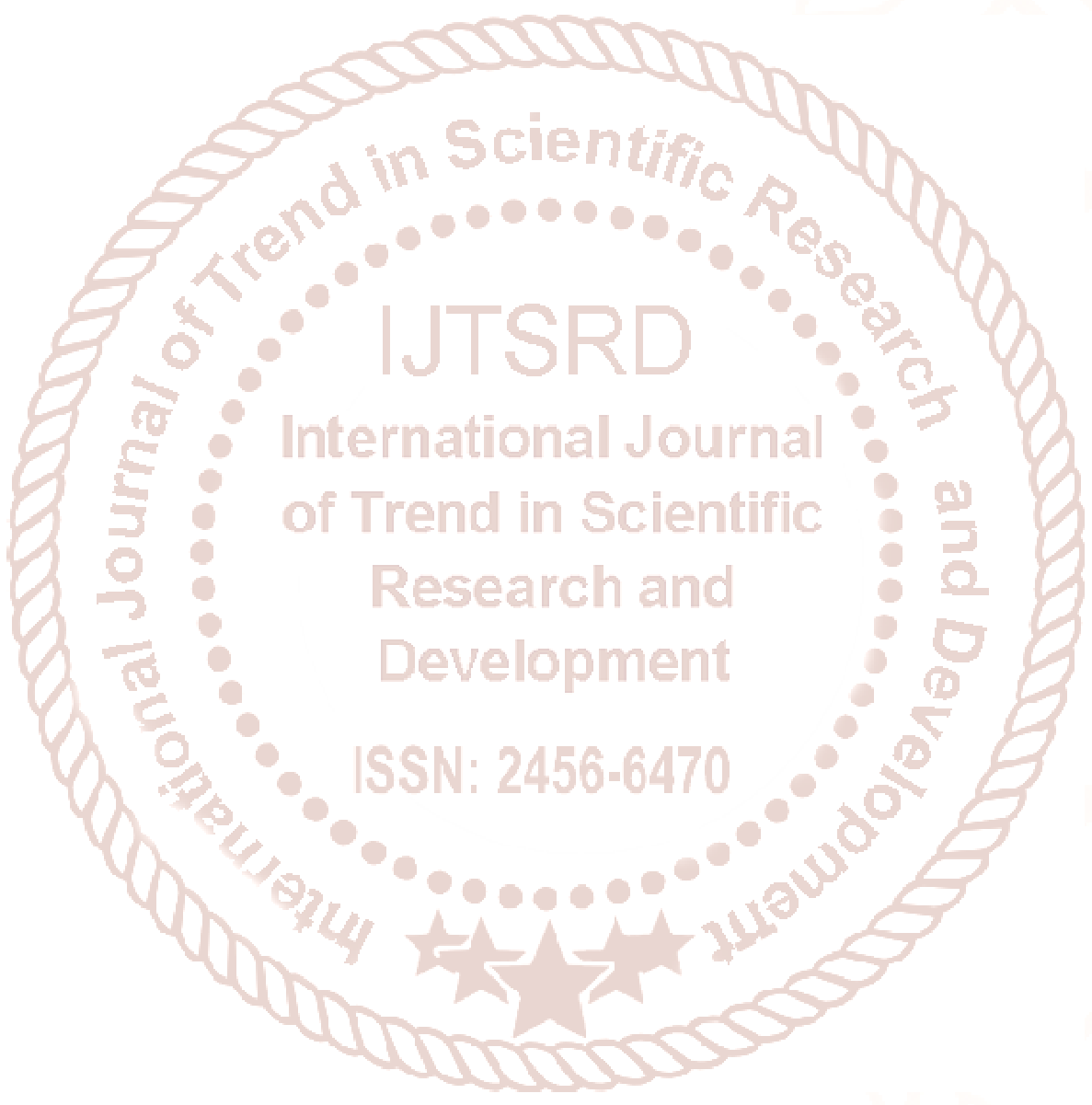

